\title{
P6: Altersgerechtes Personaleinsatzmanagement in der Pflege
}

\author{
Karl Blum
}

Online publiziert: 23. Oktober 2013

(C) Springer-Verlag Wien 2013

Einleitung: Angesichts des demografischen Wandels und des Fachkräftemangels in der Pflege ist es für die Krankenhäuser zusehends wichtiger, Pflegekräfte durch altersgerechte Arbeitsbedingungen langfristig an das Krankenhaus zu binden. Ein Kernelement des altersgerechten Arbeitens im Krankenhaus ist das Personaleinsatzmanagement. Konkret bedeutet dies, dass zum einen altersgerechte Arbeitsplätze im Krankenhaus identifiziert oder geschaffen werden. Zum anderen ist über die Personaleinsatzplanung zu gewährleisten, dass ein altersgerechter Personaleinsatz tatsächlich erfolgt oder den Mitarbeitern zumindest entsprechende Optionen systematisch kommuniziert und offeriert werden.

Methodik: Mittels Fallstudien in ausgewählten Best-Practice-Krankenhäusern wurden innovative Maßnahmen und Konzepte für einen längerfristigen Verbleib im Beruf identifiziert. Methodisch kamen in den Fallstudien vor allem qualitative Interviews, standardisierte Mitarbeiterbefragungen und Dokumentenanalysen zum Einsatz.

Ergebnisse: In den Fallstudien konnten fünf zentrale Modelle eines altersgerechten Personaleinsatzmanagements identifiziert werden:
- Pflegeinterne Stellenwechsel

- Job-Rotation

- Altersgerechte Arbeitsverteilung auf den Stationen

- Stellenwechsel in neue Tätigkeitsfelder im Krankenhaus

- Trägerinterne Stellenwechsel aus dem Krankenhaus

Der Beitrag stellt die Modelle dar, untersucht die jeweiligen Vorgehensweisen und beschreibt Praxisbeispiele aus den Projektkrankenhäusern.

Schlussfolgerungen: Ziel des altersgerechten Personaleinsatzmanagements ist eine ausgewogene Verteilung von Arbeitsanforderungen und Arbeitsbelastungen über die verschiedenen Lebensphasen und Altersgruppen in der Pflege. Praxisbewährung und Nachhaltigkeit der einschlägigen Modelle haben einen wichtigen Einfluss auf die Arbeitszufriedenheit und die Gesundheit der Pflegekräfte sowie einen langen Verbleib im Beruf.
K. Blum $(\bowtie)$

Leiter Geschäftsbereich Forschung

Deutsches Krankenhausinstitut e. V., Düsseldorf, Deutschland

E-Mail: karl.blum@dki.de 